\title{
Impact of autologous blood injections in treatment of mid-portion Achilles tendinopathy: double blind randomised controlled trial
}

\author{
(®) $(\Theta)$ OPEN ACCESS
}

\author{
Kevin J Bell sports and exercise medicine registrar ${ }^{1}$, Mark L Fulcher sports and exercise medicine \\ physician $^{2}$, David S Rowlands senior lecturer ${ }^{3}$, Ngaire Kerse professor ${ }^{2}$
}

${ }^{1}$ Sydney Sports Medicine Centre, Sydney Olympic Park, NSW 2127, Australia; ${ }^{2}$ Department of General Practice and Primary Health Care, School of Population Health, Faculty of Medical and Health Sciences, University of Auckland, New Zealand ; ${ }^{3}$ School of Sport and Exercise, Massey University, Wellington, New Zealand

\begin{abstract}
Objective To assess the effectiveness of two peritendinous autologous blood injections in addition to a standardised eccentric calf strengthening programme in improving pain and function in patients with mid-portion Achilles tendinopathy.

Design Single centre, participant and single assessor blinded, parallel group, randomised, controlled trial.

Setting Single sports medicine clinic in New Zealand.

Participants 53 adults (mean age 49, 53\% men) with symptoms of unilateral mid-portion Achilles tendinopathy for at least three months. Participants were excluded if they had a history of previous Achilles tendon rupture or surgery or had undergone previous adjuvant treatments such as injectable therapies, glyceryl trinitrate patches, or extracorporeal shockwave therapy.
\end{abstract}

Interventions All participants underwent two unguided peritendinous injections one month apart with a standardised protocol. The treatment group had $3 \mathrm{~mL}$ of their own whole blood injected while the control group had no substance injected (needling only). Participants in both groups carried out a standardised and monitored 12 week eccentric calf training programme. Follow-up was at one, two, three and six months.

Main outcome measures The primary outcome measure was the change in symptoms and function from baseline to six months with the Victorian Institute of Sport Assessment-Achilles (VISA-A) score. Secondary outcomes were the participant's perceived rehabilitation and their ability to return to sport.

Results 26 participants were randomly assigned to the treatment group and 27 to the control group. In total, 50 (94\%) completed the six month study, with 25 in each group. Clear and clinically worthwhile improvements in the VISA-A score were evident at six months in both the treatment (change in score 18.7, 95\% confidence interval 12.3 to $25.1)$ and control $(19.9,13.6$ to 26.2$)$ groups. The overall effect of treatment was not significant $(P=0.689)$ and the $95 \%$ confidence intervals at all points precluded clinically meaningful benefit or harm. There was no significant difference between groups in secondary outcomes or in the levels of compliance with the eccentric calf strengthening programme. No adverse events were reported.

Conclusion The administration of two unguided peritendinous autologous blood injections one month apart, in addition to a standardised eccentric training programme, provides no additional benefit in the treatment of mid-portion Achilles tendinopathy.

Trial registration Australian New Zealand Clinical Trials Registry ACTRN12610000824066, WHO U1111-1117-2641.

\section{Introduction}

Achilles tendinopathy causes chronic localised pain and stiffness. It affects over half of middle distance runners ${ }^{1}$ and is responsible for up to $16 \%$ of affected people having to stop participating in their sport. ${ }^{2}$ It is also a problem among non-athletes, with $31 \%$ of cases occurring in sedentary individuals. ${ }^{3}$ The mid-portion is the most commonly affected part of the tendon. It is now recognised that the histopathological changes of tendinopathy are consistent with mucoid degeneration and poor tissue healing, rather than inflammation. ${ }^{4-6}$ Several intrinsic and extrinsic factors have been proposed, but the aetiology of this condition remains unclear. ${ }^{4-7}$ Recent studies indicate that a person's genetic makeup might predispose them to Achilles tendinopathy. ${ }^{8-10}$

Numerous different methods have been proposed to treat tendinopathy, such as eccentric training, the injection of corticosteroids and sclerosants, prolotherapy, extracorporeal shockwave therapy, and glyceryl trinitrate patches. ${ }^{11-13}$ Unfortunately, few of these treatments have shown consistent effectiveness and the evidence for most treatment options is sparse. The lack of prospective or randomised studies comparing treatment options makes evaluating their effectiveness difficult. 
Currently, eccentric training is the first choice of treatment of most practitioners ${ }^{14}$ although the methodological quality of the studies suggesting efficacy has recently been questioned. ${ }^{15} 16$

Autologous blood injections involve the reinjection of a patient's own whole blood and are a relatively novel and increasingly popular option for the treatment of tendinopathy. The proposed mechanism of action is that the cytokines and growth factors within the injected blood help to stimulate tissue healing and production of type 1 collagen. ${ }^{17-19} \mathrm{~A}$ few recent papers have suggested that such injections are a useful management option in the treatment of tendinopathy, ${ }^{19-23}$ but only one study, which has limitations, examined their use in the treatment of Achilles tendinopathy. ${ }^{24} \mathrm{We}$ investigated the efficacy of autologous blood injections as a treatment option in addition to an eccentric training programme in improving pain and function in those with mid-portion Achilles tendinopathy.

\section{Methods}

This study was a prospective, participant and single assessor blinded, two arm, randomised controlled trial with 1:1 treatment allocation.

\section{Participants}

Participants were eligible for inclusion if they were aged over 18 and presenting with their first episode of mid-portion Achilles tendinopathy. Symptoms must have been present for at least three months and the diagnosis had to be confirmed by diagnostic ultrasonography. An experienced musculoskeletal radiologist carried out the ultrasound scans (Philips iU22 machine with L17-5 linear array transducer 5-17 MHz; Bothell, Washington, DC). The findings were graded as mild if the tendon displayed only fusiform thickening, moderate if there were additional hypoechoic areas present, and severe if neovessels were also present on Doppler scanning. We excluded patients with bilateral Achilles tendon symptoms, alternative diagnoses such as insertional Achilles tendinopathy, or previous Achilles tendon rupture or surgery or who had previously undergone adjuvant therapies such as any kind of injection, glyceryl trinitrate patches, or extracorporeal shockwave therapy. We did not exclude people who had undertaken some form of eccentric training.

This study was conducted at a specialist multidisciplinary sports medicine clinic (Wakefield Sportsmed, Wellington, New

Zealand). Participants were recruited after letters were sent to over 200 general practitioners, physiotherapists, osteopaths, and podiatrists in the Wellington region, articles were published in a number of local newspapers and websites, or direct referral. People who were interested in taking part gave written informed consent and were evaluated with a standardised procedure to collect baseline data including sociodemographic data and information on sports they participated in. Participants were recruited between November 2010 and April 2011 and followed up for six months.

\section{Interventions}

All participants received two unguided peritendinous injections at the site of maximal tenderness as marked by the blinded assessor. The participants lay prone with a screen over their legs to block any view of the intervention taking place. One of three non-blinded sports physicians administered the injections at baseline and one month later. All participants had $3 \mathrm{~mL}$ of their own blood taken from their antecubital fossa. A standardised three pass injection technique through a single puncture site was used for participants in both randomised groups. The needle was firstly inserted perpendicular to the tendon, with a second pass aimed $20^{\circ}$ superiorly in the plane of the tendon, and a third pass aimed $20^{\circ}$ inferiorly. In the treatment group, patients received the $3 \mathrm{~mL}$ of blood injected during the three passes, with $1 \mathrm{~mL}$ injected during each pass. The control group underwent dry needling with the same technique but no substance was injected. The blood was not processed, and no local anaesthetic was used. After the injection, the puncture site was dressed and all participants were supervised while they massaged the area for five minutes followed by a five minute walk.

All participants were instructed to rest from physical activity until any discomfort from the injection had settled down. They were given written, pictorial, and verbal instructions about how to carry out the standardised eccentric training programme. ${ }^{25}$ This programme required each participant to do 180 eccentric heel drops over a step each day for a minimum of 12 weeks on the injured leg only. Participants were allowed to exercise during this time as long as it did not induce pain rated more than 4 on a 10 point scale.

\section{Outcomes}

The primary outcome measure was the Victorian Institute of Sports Assessment-Achilles (VISA-A) score measured at baseline and again at one, two, three, and six months. ${ }^{2}$ The VISA-A score is a validated measure of pain and function in those with Achilles tendinopathy. Scores range from 0-100, with 100 indicating a symptom free tendon. Each participant completed the questionnaire under supervision from the blinded assessor. Our primary outcome was the difference in the change in VISA-A score from baseline between the groups.

Secondary outcomes included a six point Likert score at final follow-up to assess perceived rehabilitation. All participants were asked to rate their level of return to sport at the time of final follow-up. All participants completed daily eccentric training compliance logs recording the number of repetitions and the amount of any additional weight carried.

\section{Sample size}

The final sample size was estimated with a continuous variables method for studies comparing two group means, with a type I error rate $(\alpha)$ of 0.05 and power of $0.80(\beta=0.20) .{ }^{26}$ While consensus in the literature has not yet been reached as to the minimally clinically important change in VISA-A score, ${ }^{27}$ we settled on a change score of 10 based on the primary criteria that this effect size would represent a noticeable functional difference in the participant's ability to participate in sport (questions 7 and 8 of the VISA-A questionnaire). We used an SD of 17, drawn from a VISA-A reliability study, which resulted in an estimated sample size of 24 per group. ${ }^{2}$ The allowance of a $10 \%$ loss to follow-up brought the estimate to 26 per group. During the peer review process, we noted an error in the sample size calculation as the SD used reflected an overall VISA-A score rather than a change in score over time. Subsequent estimates resulted in a sample size per group ranging from 11 to 18 , suggesting the study was adequately powered to support the conclusions drawn.

\section{Randomisation}

Equal numbers of opaque envelopes containing treatment group and control group were shuffled and kept together in a box. Each participant underwent simple randomisation into one of the two groups by selecting a sealed envelope from the box. 
The participant's name and enrolment number were written on the envelope. After all baseline assessments were completed, the non-blinded doctor carrying out the intervention opened the envelope to see the group allocation. Allocation remained concealed from both the participant and the assessor, who remained blinded throughout the study. At final follow-up all participants completed a blinding analysis questionnaire to determine if blinding had been compromised.

\section{Statistical analysis}

Analysis was carried out with statistical software (SPSS version 19.0, 2010, SPSS Science, Chicago, IL). The interaction between treatment and time was treated in a linear model with repeated measures to examine the effect of eccentric exercise and autologous blood injection on change in the VISA-A scores over six months' follow up and to provide the point estimates and uncertainty ( $95 \%$ confidence intervals) relative to baseline. We also evaluated the effect of adjustment for age, sex, ethnicity, level of physical activity, duration of symptoms, severity on ultrasonography, compliance with eccentric training, additional weight carried during eccentric training, and eccentric exercise technique on the magnitude of the effect of treatment on the change in VISA-A score. The secondary

outcomes-participant's ability to return to sport and perceived degree of rehabilitation - were analysed with one way analysis of variance. We used intention to treat analysis via last observation carried forward for the three participants lost to follow-up, with their final recorded outcome being brought forward for the remaining missed data points.

\section{Results}

\section{Recruitment}

Of 88 people considered for recruitment, 53 (95\% participation rate) met the inclusion criteria and chose to enrol in the study. Of these, 50 (94\%) completed all follow-up. Figure 1 shows the flow of participants through the study $\Downarrow$. Table 1 shows the baseline characteristics of the participants in each of the two groups $\Downarrow$.

\section{Primary outcomes}

The treatment group's mean VISA-A score improved from 58.1 at baseline to 76.8 at six months; an overall change of 18.7 (95\% confidence interval 12.3 to 25.1 ). The control group improved from 57.3 to 77.2 ; an overall change of 19.9 (13.6 to 26.2). Figure $2 \Downarrow$ shows the absolute mean VISA-A scores of both groups throughout the study, and table $2 \Downarrow$ shows the estimates and uncertainty of the mean change in scores over time. Clinically worthwhile improvements in mean VISA-A score were evident by six months in both groups (table 2). The overall effect of treatment, however, was not significant $(\mathrm{P}=0.689)$ and the $95 \%$ uncertainty at all points precluded clinically meaningful benefit or harm. Adjustment for the effect of each of the covariates had no impact on the final outcome relative to the unadjusted effect. Sensitivity analyses of the impact of the last observation carried forward on the primary outcome, both with and without imputation of missing data, did not alter our conclusions.

\section{Secondary outcomes}

There was no significant difference at final follow-up in the participant's perceived rehabilitation according to the Likert score $(\mathrm{P}=0.881)$ or of their ability to return to their desired sport ( $\mathrm{P}=0.679)$ (tables $3 \Downarrow$ and $4 \Downarrow$ ).
Mean compliance with eccentric repetition was similar in the treatment and control groups, 65\% (SD 17\%) and 62\% (SD $24 \%)$, respectively $(\mathrm{P}=0.612)$. Similarly, the mean amount of weight added to a backpack worn while carrying out the eccentric training was no different between the groups $(\mathrm{P}=0.591)$, with $8.8 \mathrm{~kg}$ (SD 9.8) and 10.5 kg (SD 12.1) being carried in the treatment and control groups.

Of the 50 participants who completed follow-up, 49 answered the question "Which group do you think you were allotted to?" before the unblinding of their group allocation. The one participant who did not answer this question had inadvertently overlooked it. There was no significant difference between the predictions of those in each of the groups $(\mathrm{P}=0.564)$, with over half of the participants in each group answering that they were unsure into which group they had been randomised.

\section{Adverse events}

There were no complications or adverse events as a result of the injections carried out during the study.

\section{Discussion}

\section{Summary of principal findings}

To our knowledge, this is the first double blinded, randomised, controlled trial investigating the efficacy of autologous blood injections as a treatment for mid-portion Achilles tendinopathy in addition to a standardised eccentric training programme. Both groups showed a steady and clinically meaningful improvement in the mean VISA-A scores throughout the study. The addition of two peritendinous autologous blood injections administered one month apart, however, was unlikely to have resulted in additional clinically meaningful improvements in pain or in ability to return to activity.

\section{Comparisons with the literature}

The only other available published study investigating the use of autologous blood injections to treat Achilles tendinopathy concluded that injections produced a moderate improvement in symptoms. ${ }^{24}$ This previous study lacked blinding and allocation concealment, administered a variable number of injections, included participants with unilateral and bilateral symptoms, and had a high dropout rate. The sample size recruited for our study was large enough to preclude possible clinical benefits and harm and was also similar to that in other randomised trials that used the change in VISA-A score as the primary outcome measure (40-75 participants). ${ }^{24-29}$ The monitoring of eccentric training was also similar to that used by some other investigators, ${ }^{28}{ }^{30}$ though others did not mention steps taken to monitor compliance. ${ }^{29}{ }^{31}$ Good compliance probably contributes to a participant's clinical improvement. ${ }^{25}$ Without accurate monitoring, any benefit attributable to the intervention might actually be caused by disparity between groups in the compliance with eccentric training or would at least increase the variation of the results. It is also likely that there would be increased variation in measurement between participants.

\section{Explanations and clinical implications}

The use of autologous blood injections to treat tendinopathy has become particularly popular in recent years, despite the apparent lack of quality evidence supporting their use. ${ }^{19-33}$ Our findings suggest that the application of autologous blood injections as described has no clear clinically beneficial effect, and based on this finding we cannot recommend their future use. There are, however, several other possible explanations for 
the outcome. Firstly, the needling process itself might have created sufficient bleeding in the control participants to induce a healing response. Secondly, administering injections closer together would have enhanced any potential benefit from maintaining a steady state effect of growth factors present in the blood. ${ }^{17}{ }^{19}$ The lack of ultrasound guidance, or the injections being directed peritendinously rather than intratendinously, could have affected the outcome. From our experience however, most autologous blood injections administered in New Zealand are delivered peritendinously. Several reports of benefit from unguided injections in tendinopathy support this type of administration. ${ }^{24-36}$ There are also numerous studies indicating that peritendinous injections of differing substances can provide benefit in the treatment of Achilles tendinopathy. ${ }^{24-38}$ The intratendinous injection of platelet rich plasma, a derivative of whole blood, has been investigated in the treatment of mid-portion Achilles tendinopathy with mixed results to date. ${ }^{39}$ The proposed mechanism of action of autologous blood injections and platelet rich plasma is similar, but the former is cheaper as it does not require specific preparation and a smaller volume of blood is taken from participants. The negligible clinical effect of autologous blood injections in this study, and the mixed results of platelet rich plasma, suggest that these forms of injection therapy are not useful for the treatment of mid-portion Achilles tendinopathy. Eccentric training is already recognised as a good treatment option for Achilles tendinopathy, ${ }^{15-28}$ and both groups showed a clinical improvement as a result of the eccentric training component. It was therefore difficult to show an additive clinically meaningful effect of autologous blood injections. We did not use any type of substance in the injections in the control group, whereas most other studies used injections of normal saline $e^{28-42}$ or local anaesthetic. ${ }^{43} 44$ There is recent evidence to suggest that higher levels of extracellular sodium suppress transient receptor potential V1 (TRPV1) activity, a non-selective cation channel present in nociceptors that helps to integrate pain perception. ${ }^{45}$ We considered that injecting normal saline could itself result in reduced pain sensation, and as such, not strictly act as a control intervention. Indeed, the group allocation prediction analysis shows that participants were unable to determine which group that they had been randomised to, suggesting that with the use of our control intervention, we were able to maintain adequate blinding.

\section{Strengths and limitations of the study}

Strengths of this study were the high participation rate (95\%) and low dropout rate $(6 \%)$. The participants reflected a wide range of society with ages ranging from 27 to 76 and sex being evenly matched with $53 \%$ men and $47 \%$ women. There was, however, an under-representation of minority ethnic groups, with $91 \%$ of the participants identifying themselves as European and the $9 \%$ remaining as New Zealand Maori. A further strength was the use of the validated primary outcome measure, the VISA-A questionnaire, which was completed in the presence of the single blinded assessor. We also used daily monitoring of eccentric training compliance with a written $\log$, which could have encouraged good compliance and showed that there were no differences between the groups in the eccentric training aspect of the management.

\section{Future research}

We conclude that the administration of unguided peritendinous autologous blood injections does not confer an additional benefit to rehabilitation outcomes resulting from a standardised eccentric training programme. Follow-up studies could involve the injections being given intratendinously under ultrasound guidance, a shorter time interval between injections, or an increase in the volume of injected blood. It would also be beneficial to have a third treatment group who undertake an eccentric training programme only, without injection. While such derivations would aid in estimation of the effect of the needling itself, it would be without blinding and therefore susceptible to placebo effects. A fourth treatment with autologous blood injections in the absence of eccentric training would help to determine whether any benefit is obtained from injections in isolation.

We thank lan Murphy and Jake Pearson for assistance carrying out the interventions; lan Best and Pacific Radiology for carrying out the ultrasound scans; Jessica Thomas, Simon Moyes, and Avinesh Pillai for assistance with the data analysis: Lyn Kimber, Louise Caig, and Ruth Highet for assistance with administrative and logistical support; and all the participants for their time and efforts during the trial.

Contributors: KJB, MLF, DSR, and NK all participated in designing the study. KJB was the principal study investigator and drafted the manuscript. The authors all had free access to the complete data set and actively participated in the analysis and interpretation of results, critically revised the article and approved the final manuscript for publication. KJB is guarantor.

Funding: This research received no specific grant from any funding agency in the public, commercial, or not-for-profit sectors.

Competing interests: All authors have completed the ICMJE uniform disclosure form at www.icmje.org/coi_disclosure.pdf (available on request from the corresponding author) and declare: no support from any organisation for the submitted work; no financial relationships with any organisations that might have an interest in the submitted work in the previous three years; no other relationships or activities that could appear to have influenced the submitted work.

Ethical approval: This study was approved by the New Zealand Ministry of Health, Central regional ethics committee on 21 September 2010 (reference No CEN/10/07/029) and informed consent was given by all patients.

Data sharing: Technical appendix, statistical code, and dataset are available from the corresponding author.

1 Zafar MS, Mahmood A, Maffulli N. Basic science and clinical aspects of achilles tendinopathy. Sports Med Arthrosc 2009;17:190-7.

2 Robinson JM, Cook JL, Purdam C, Visentini PJ, Ross J, Maffulli N, et al. The VISA-A questionnaire: a valid and reliable index of the clinical severity of Achilles tendinopathy. Br J Sports Med 2001;35:335-41.

3 Rolf C, Movin T. Etiology, histopathology, and outcome of surgery in achillodynia. Foot Ankle Int 1997; 18:565-9.

4 Alfredson $\mathrm{H}$. Conservative management of Achilles tendinopathy: new ideas. Foot Ankle Clin 2005; 10:321-9.

5 Astrom M, Rausing A. Chronic Achilles tendinopathy. A survey of surgical and histopathologic findings. Clin Orthop 1995;316:151-64.

6 Leadbetter WB. Anti-inflammatory therapy in sports injury. The role of nonsteroidal drugs and corticosteroid injection. Clin Sports Med 1995;14:353-410.

7 Rasmussen S, Christensen M, Mathiesen I, Simonson O. Shockwave therapy for chronic Achilles tendinopathy: a double-blind, randomized clinical trial of efficacy. Acta Orthop 2008;79:249-56.

8 Raleigh SM, van der Merwe L, Ribbans WJ, Smith RKW, Schwellnus MP, Collins M. Variants within the MMP3 gene are associated with Achilles tendinopathy: possible interaction with the COL5A1 gene. Br J Sports Med 2009;43:514-20.

9 Mokone GG, Schwellnus MP, Noakes TD, Collins M. The COL5A1 gene and Achilles tendon pathology. Scand J Med Sci Sports 2006;16:19-26.

10 September AV, Cook J, Handley CJ, van der Merwe L, Schwellnus MP, Collins M. Variants within the COL5A1 gene are associated with Achilles tendinopathy in two populations. Br J Sports Med 2009;43:357-65.

11 Lake JE, Ishikawa SN. Conservative treatment of Achilles tendinopathy: emerging techniques. Foot Ankle Clin 2009;14:663-74.

12 Longo UG, Ronga M, Maffulli N. Achilles tendinopathy. Sports Med Arthrosc 2009;17:112-26.

13 Abbassian A, Khan R. Achilles tendinopathy: pathology and management strategies. $\mathrm{Br}$ $J$ Hosp Med 2009;70:519-23.

14 Alfredson H. Chronic midportion Achilles tendinopathy: an update on research and treatment. Clin Sports Med 2003;22:727-41.

15 Magnussen RA, Dunn WR, Thomson AB. Nonoperative treatment of midportion Achilles tendinopathy: a systematic review. Clin J Sport Med 2009;19:54-64.

16 Kingma JJ, de Knikker R, Wittink HM, Takken T. Eccentric overload training in patients with chronic Achilles tendinopathy: a systematic review. Br J Sports Med 2007;41:e3. 


\section{What is already know on this topic}

Several studies have suggested that the injection of autologous blood can be helpful in the treatment of various tendinopathies Autologous blood injections are a relatively common treatment, despite a lack of high quality evidence supporting their use in the management of Achilles tendinopathy

\section{What this study adds}

The use of peritendinous autologous blood injections does not reduce pain or improve function in those with mid-portion Achilles tendinopathy when they are combined with an eccentric training programme

17 Iwasaki M, Nakahara H, Nakata K, Nakase T, Kimura T, Ono K. Regulation of proliferation and osteochondrogenic differentiation of periosteum-derived cells by transforming growth factor-beta and basic fibroblast growth factor. J Bone Joint Surg Am 1995;77:543-54.

18 Kajikawa Y, Morihara T, Sakamoto H, Matsuda K-i, Oshima Y, Yoshida A, et al. Platelet-rich plasma enhances the initial mobilization of circulation-derived cells for tendon healing. $J$ Cell Physiol 2008;215:837-45.

19 Connell DA, Ali KE, Ahmad M, Lambert S, Corbett S, Curtis M. Ultrasound-guided autologous blood injection for tennis elbow. Skeletal Radiol 2006;35:371-7.

20 Suresh SP, Ali KE, Jones H, Connell DA. Medial epicondylitis: is ultrasound guided autologous blood injection an effective treatment? Br J Sports Med 2006;40:935-9.

21 Thanasas C, Papadimitriou G, Charalambidis C, Paraskevopoulos I, Papanikolaou A. Platelet-rich plasma versus autologous whole blood for the treatment of chronic lateral elbow epicondylitis: a randomized controlled clinical trial. Am J Sports Med 2011;39:2130-4.

22 Creaney L, Wallace A, Curtis M, Connell D. Growth factor-based therapies provide additional benefit beyond physical therapy in resistant elbow tendinopathy: a prospective, single-blind, randomised trial of autologous blood injections versus platelet-rich plasma injections. Br J Sports Med 2011:45:966-71.

23 James SLJ, Ali K, Pocock C, Robertson C, Walter J, Bell J, et al. Ultrasound guided dry needling and autologous blood injection for patellar tendinosis. Br J Sports Med 2007;41:518-22.

24 Pearson J, Rowlands D, Highet R. Autologous blood injection to treat achilles tendinopathy? A randomized controlled trial. J Sport Rehabil 2012;21:218-24

25 Alfredson $\mathrm{H}$, Pietila T, Jonsson P, Lorentzon R. Heavy-load eccentric calf muscle training for the treatment of chronic Achilles tendinosis. Am J Sports Med 1998;26:360-6.

26 Snedecor GW, Cochran WG. Statistical methods. 8th ed. lowa State Press, 1989.

27 Iversen JV, Bartels EM, Langberg H. The Victorian Institute of Sports Assessment Achilles questionnaire (VISA-A)-a reliable tool for measuring Achilles tendinopathy. Int J Sports Phys Ther 2012;7:76-84.

28 de Vos RJ, Weir A, van Schie HTM, Bierma-Zeinstra SMA, Verhaar JAN, Weinans $\mathrm{H}$, et al. Platelet-rich plasma injection for chronic Achilles tendinopathy: a randomized controlled trial. JAMA 2010;303:144-9.

29 Rompe JD, Nafe B, Furia JP, Maffulli N. Eccentric loading, shock-wave treatment, or a wait-and-see policy for tendinopathy of the main body of tendo Achillis: a randomized controlled trial. Am J Sports Med 2007;35:374-83.

30 de Vos RJ, Weir A, Visser RJA, de Winter T, Tol JL. The additional value of a night splint to eccentric exercises in chronic midportion Achilles tendinopathy: a randomised controlled trial. Br J Sports Med 2007;41:e5.

31 Rompe JD, Furia JP, Maffulli N. Mid-portion Achilles tendinopathy-current options for treatment. Disabil Rehabil 2008;30:1666-76.

32 Ozturan KE, Yucel I, Cakici H, Guven M, Sungur I. Autologous blood and corticosteroid injection and extracoporeal shock wave therapy in the treatment of lateral epicondylitis. Orthopedics 2010;33:84-91.

33 Kazemi M, Azma K, Tavana B, Rezaiee Moghaddam F, Panahi A. Autologous blood versus corticosteroid local injection in the short-term treatment of lateral elbow tendinopathy: a randomized clinical trial of efficacy. Am J Phys Med Rehabil 2010;89:660-7.
34 Bisset L, Beller E, Jull G, Brooks P, Darnell R, Vicenzino B. Mobilisation with movement and exercise, corticosteroid injection, or wait and see for tennis elbow: randomised trial. BMJ 2006;333:939.

35 Edwards SG, Calandruccio JH. Autologous blood injections for refractory lateral epicondylitis. J Hand Surg Am 2003;28:272-8.

36 Orchard J, Massey A, Brown R, Cardon-Dunbar A, Hofmann J. Successful management of tendinopathy with injections of the MMP-inhibitor aprotinin. Clin Orthop 2008;466:1625-32

37 Fredberg U, Bolvig L, Pfeiffer-Jensen M, Clemmensen D, Jakobsen BW, Stengaard-Pedersen K. Ultrasonography as a tool for diagnosis, guidance of local steroid injection and, together with pressure algometry, monitoring of the treatment of athletes with chronic jumper's knee and Achilles tendinitis: a randomized, double-blind, placebo-controlled study. Scand J Rheumatol 2004;33:94-101.

38 Yelland MJ, Sweeting KR, Lyftogt JA, Ng SK, Scuffham PA, Evans KA. Prolotherapy injections and eccentric loading exercises for painful Achilles tendinosis: a randomised trial. Br J Sports Med 2011;45:421-8.

39 Gaweda K, Tarczynska M, Krzyzanowski W. Treatment of Achilles tendinopathy with platelet-rich plasma. Int J Sports Med 2010;31:577-83.

40 Brown R, Orchard J, Kinchington M, Hooper A, Nalder G. Aprotinin in the management of Achilles tendinopathy: a randomised controlled trial. Br J Sports Med 2006;40:275-9.

41 De Jonge S, de Vos RJ, Weir A, van Schie HTM, Bierma-Zeinstra SMA, Verhaar JAN, et al. One-year follow-up of platelet-rich plasma treatment in chronic Achilles tendinopathy: a double-blind randomized placebo-controlled trial. Am J Sports Med 2011:39:1623-9.

42 Wolf JM, Ozer K, Scott F, Gordon MJV, Williams AE. Comparison of autologous blood, corticosteroid, and saline injection in the treatment of lateral epicondylitis: a prospective, randomized, controlled multicenter study. J Hand Surg Am 2011;36:1269-72.

43 Zeisig E, Fahlstrom M, Ohberg L, Alfredson $\mathrm{H}$. Pain relief after intratendinous injections in patients with tennis elbow: results of a randomised study. Br J Sports Med 2008;42:267-71.

44 Mishra A, Pavelko T. Treatment of chronic elbow tendinosis with buffered platelet-rich plasma. Am J Sports Med 2006;34:1774-8.

45 Ohta T, Imagawa T, Ito S. Novel gating and sensitizing mechanism of capsaicin receptor (TRPV1): tonic inhibitory regulation of extracellular sodium through the external protonation sites on TRPV1. J Biol Chem 2008;283:9377-87.

\section{Accepted: 26 March 2013}

\section{Cite this as: BMJ 2013;346:f2310}

This is an Open Access article distributed in accordance with the Creative Commons Attribution Non Commercial (CC BY-NC 3.0) license, which permits others to distribute, remix, adapt, build upon this work non-commercially, and license their derivative works on different terms, provided the original work is properly cited and the use is non-commercial. See: http://creativecommons.org/licenses/by-nc/3.0/. 


\section{Tables}

Table 1| Baseline characteristics of patients with mid-portion Achilles tendinopathy according to allocation to autologous blood injections (treatment) or dry needling (control). Figures are numbers (percentage) of participants unless stated otherwise

\begin{tabular}{lcc} 
Characteristics & Treatment (n=26) & Control (n=27) \\
Mean (SD) age (years) & $51.2(10.6)$ & $47.2(9.7)$ \\
\hline Men & $16(62)$ & $12(44)$ \\
\hline European & $24(92)$ & $24(89)$ \\
\hline Left side affected & $20(77)$ & $13(48)$ \\
\hline Mean (SD) duration of symptoms (months) & $22.9(33.1)$ & $38.6(84.6)$ \\
\hline Mean (SD) duration of symptoms (months) (in those with duration $\leq 100$ months) & $14.8(17.4)$ & $17.7(20.1)$ \\
\hline Participates in physical activity/sport & $22(85)$ & $27(100)$ \\
\hline Physical activity: & & \\
\hline No change & $12(46)$ & $9(33)$ \\
\hline Reduced & $9(35)$ & $7(26)$ \\
\hline Ceased & $5(19)$ & $11(41)$ \\
\hline Changes on ultrasonography: & & \\
\hline Severe & $5(19)$ & $1(4)$ \\
\hline Moderate & $16(62)$ & $17(63)$ \\
\hline Mild & $5(19)$ & $9(33)$ \\
\hline Mean (SD) VISA-A score & $58.1(\mathrm{SD} 17.2)$ & $57.3(\mathrm{SD} 12.7)$ \\
\hline
\end{tabular}


Table 2| Mean change (95\% confidence interval) in VISA-A scores from baseline in patients with mid-portion Achilles tendinopathy according to allocation to autologous blood injections (treatment) or dry needling (control)

\begin{tabular}{lccc} 
& Treatment & Control & Between group difference $(95 \% \mathrm{Cl})$ \\
Month 1 & $7.9(3.8$ to 12.1$)$ & $5.3(0.9$ to 9.8$)$ & $2.6(-3.4$ to 8.6$)$ \\
\hline Month 2 & $12.0(6.4$ to 17.6$)$ & $8.6(3.7$ to 13.4$)$ & $3.4(-3.8$ to 11.0$)$ \\
\hline Month 3 & $15.2(8.8$ to 21.5$)$ & $14.9(9.3$ to 20.4$)$ & $0.3(-6.9$ to 7.5$)$ \\
\hline Month 6 & $18.7(12.3$ to 25.1$)$ & $19.9(13.6$ to 26.2$)$ & $-1.2(-10.0 \text { to } 7.9)^{\star}$ \\
\hline
\end{tabular}

${ }^{*} \mathrm{P}=0.689$ for regression with repeated measures for difference between groups over time. 
Table 3| Likert scores of perceived rehabilitation at six month follow-up in patients with mid-portion Achilles tendinopathy according to allocation to autologous blood injections (treatment) or dry needling (control). Figures are numbers (percentage) of participants

\begin{tabular}{lcc} 
Likert score & Treatment $(\mathbf{n = 2 5 )}$ & Control $(\mathbf{n}=\mathbf{2 5})$ \\
\hline 1 (completely recovered) & $10(40)$ & $9(36)$ \\
\hline 2 (much better) & $13(52)$ & $13(52)$ \\
\hline 3 (a little better) & $2(8)$ & $3(12)$ \\
\hline 4-6 (unchanged/much worse) & 0 & 0
\end{tabular}


Table 4| Ability to return to sport at six month follow-up in patients with mid-portion Achilles tendinopathy according to allocation to autologous blood injections (treatment) or dry needling (control). Figures are numbers (percentage) of participants

\begin{tabular}{lcc} 
Not normally active in sport & Treatment $(\mathbf{n}=\mathbf{2 5})$ & Control $(\mathbf{n}=\mathbf{2 5})$ \\
\hline No return to sport & $7(28)$ & $1(4)$ \\
\hline Returned to sport, but not in desired sport & $1(4)$ & $1(4)$ \\
\hline Returned to desired sport, but not to pre-injury level & $3(12)$ & $10(16)$ \\
\hline Returned to pre-injury level in desired sport & $13(52)$ & $9(36)$ \\
\hline
\end{tabular}




\section{Figures}

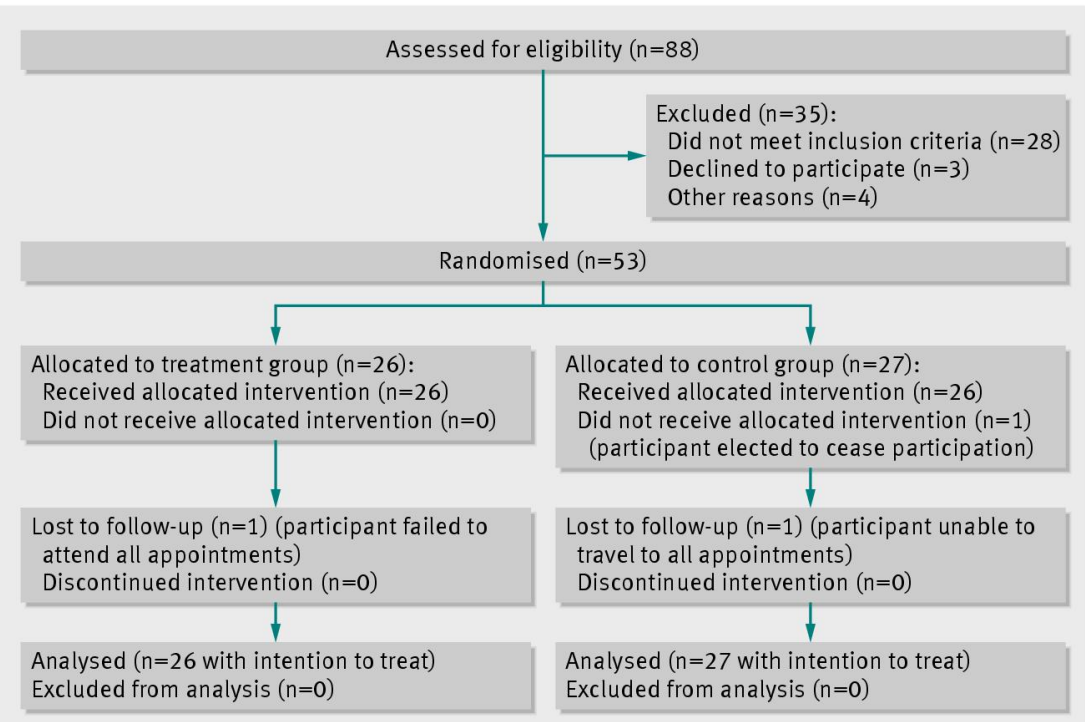

Fig 1 Flow of participants with mid-portion Achilles tendinopathy through study on impact of autologous blood injections

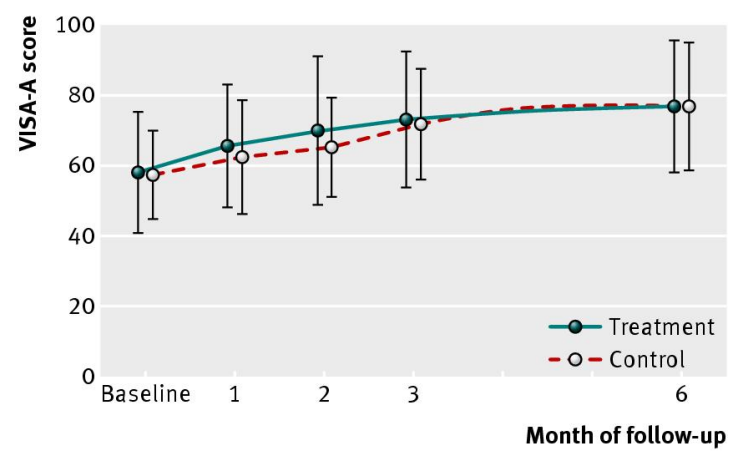

Fig 2 Absolute mean VISA-A scores over time in participants with mid-portion Achilles tendinopathy according to randomisation to autologous blood injections (treatment) or dry needling (control) 